\title{
Various Radiographic Appearances of Fibrous Dysplasia in the Mandible - A Case Report
}

\author{
Johannes Angermair ${ }^{1 *}$, Tobias Fretwurst ${ }^{1}$, Wiebke Semper-Hogg ${ }^{1}$, Gian Kayser ${ }^{2}$, Katja Nelson ${ }^{1}$ and Rainer Schmelzeisen ${ }^{1}$ \\ ${ }^{1}$ Department of Oral- and Craniomaxillofacial Surgery, University Medical Center Freiburg, Hugstetterstrasse 55, 79106 Freiburg, Germany \\ ${ }^{2}$ Institute for Clinical Pathology, University Medical Center Freiburg, Germany
}

\begin{abstract}
Introduction: Fibrous dysplasia appears in a clinically and radiologically variable way. Radiographic diagnostic is an important factor for final diagnosis especially as this rare lesion is often observed accidently in dental radiographic examinations. Therefore, the present case report demonstrates deviating clinical and radiological manifestations of monostotic and polyostotic forms of fibrous dysplasia (FD) in the facial area and its impact on dental and surgical therapy.
\end{abstract}

Presentation of case: In the first patient, showing a monostotic form a hard, non-compressive swelling in the lower incisor area was detectable and radiographic investigation showed a "ground glass"-like radiopacity in the lower mandible. A surgical reduction of the process and a biopsy were indicated due to progression of the lesion and the development of aesthetic impairment at a young age. In the second patient, with a polyostotic manifestation, radiographic investigation revealed mixed heterogeneous sclerotic areas in the left mandibular angle and a "ground glass"-like pattern and osteolyses spreading out from the sphenoid sinus. A biopsy was obtained to confirm the radiological diagnosis without complete removal of the diseased bone.

Conclusion: The two cases demonstrate the strongly varying clinical and radiological appearances of craniofacial FD and underline why it poses a challenge to the medical and dental practitioner. Furthermore, the present case report is discussing different diagnostic and therapy concepts according to different forms of FD and the consequences for dental therapy. Therapy strategies should always be determined by the progression and dimension of the disease and requires close interdisciplinary cooperation.

Keywords: Fibrous dysplasia; Craniofacial fibrous dysplasia; McCune-Albright syndrome; Cone beam computer tomography; Radiographic diagnostics

\section{Introduction}

Fibrous dysplasia (FD) is a rare, congenital malformation of the bone tissue. The disease occurs as a monostotic form, in which only a single bone is affected, and a polyostotic form involving multiple skeletal locations. Fibrous dysplasia may also occur within the context of McCune-Albright Syndrome, a severe polyostotic disease in which endocrine disorders (e.g., puberty praecox, hyperthyreodism, Cushing's disease) and pigmentation of the skin ("cafe au lait" spots) are present $[1,2]$. While fibrous dysplasia accounts for approximately $7 \%$ of all benign osseous tumors, McCune-Albright syndrome occurs very rarely, with a prevalence of $1 / 100,000$ to $1 / 1,000,000$ [3]. In 70 $80 \%$ of the cases the disease manifests in a monostatic form in the femur, tibia, and ribs, and in $20-30 \%$ as a polyostotic form [4].

The pathology is a mutation of the GNAS gene on chromosome 20 in early embryogenesis [5]. This gene encodes a G protein, which leads to dysregulation of the adenylyl cyclase [6]. Overproduction of cyclic adenosine monophosphate (cAMP) leads to a deficient differentiation of bone stem cells [7] and finally to the replacement of the healthy bone matrix by proliferating bone marrow stromal cells. This leads to the formation of the characteristic fibrous lesions $[8,9]$. Histological lesions are comprised of irregular trabecular formation within a fibrous stroma with bone marrow stromal cells without differentiation in adipocytes and osteoblasts. The bony matrix is replaced by proliferating soft tissue and shows an irregular woven structure that is described to resemble "Chinese character" pattern [8].

These changes in the bony structure lead to a characteristic radiological manifestation of the disease in juvenile patients where a "ground glass"-like radiolucency enables a tentative diagnosis. In aging, however the lesions reveal a heterogeneous appearance with focal cystic and sclerotic areas. These variable radiographic presentations impede radiographic diagnostics and require for an experienced practitioner to plan correct patient management

Therefore, the present case report demonstrates the strongly deviating clinical and radiological manifestations of monostotic and polyostotic forms of fibrous dysplasia (FD) in the facial area and its impact on dental and surgical therapy.

\section{Presentation of Case}

\section{Patient 1}

A 14-year-old male patient was admitted to the Department of Oral- and Craniomaxillofacial Surgery presenting a painless distension in the anterior lower jaw which appeared approximately 4 month before dental visit. Clinical diagnostics revealed an inconspicuous, pale reddish gingiva in the concerned region. In the right incisor region, a hard, non-compressive, non-displaceable swelling of $4 \times 3$ $\mathrm{cm}$ (Figure 1) was detectable. The patient demonstrated a conservative adequately restored juvenile dentition and good oral hygiene behavior with slightly soft plaque caused by orthognathic multiband brackets. The teeth showed neither loosening nor percussive sensitivity. In

*Corresponding author: Johannes Angermair, DMD, University Medical Center Freiburg, Hugstetterstrasse 55, D-79106 Freiburg, Germany, Tel: 0049613142111 E-mail: johannes.angermair@uniklinik-freiburg.de

Received July 11, 2017; Accepted September 18, 2017; Published September 25, 2017

Citation: Angermair J, Fretwurst T, Semper-Hogg W, Kayser G, Nelson K, et al (2017) Various Radiographic Appearances of Fibrous Dysplasia in the Mandible A Case Report. Dentistry 7: 458. doi:10.4172/2161-1122.1000458

Copyright: (c) 2017 Angermair J, et al. This is an open-access article distributed under the terms of the Creative Commons Attribution License, which permits unrestricted use, distribution, and reproduction in any medium, provided the original author and source are credited. 
addition, all teeth in the frontal lower jaw showed positive sensitivity to cold testing. Patient's ability to open the mouth was not restricted. The mentalis nerve did not demonstrate a neurological limitation by testing with two-point and sharp and numb discrimination. There was no manifestation of Vincent's Symptomatic. The general patient history was inconspicuous.

As an initial radiographic diagnostic, an orthopantomogram was taken by the transferring dentist (Figure 2). The image demonstrated an osseous, cancellous structural change in the frontal lower jaw area. A cone beam computertomography (СBCT) image, with adapted field-of-view to reduce radiation load (Figure 3), was used for threedimensional delineation of the X-ray diffraction, which showed a $4 \times$ $3 \mathrm{~cm}$, "ground glass"-like opacity in the lower mandible. Based on this characteristic appearance, a tentative diagnosis of fibrous dysplasia was made.

A sample biopsy under local anesthesia was taken via a marginal mucoperiosteal flap to confirm the findings. Since the patient desired an aesthetic correction, a biopsy with a trepan drill in the chin region was made, the protrusion in the frontal lower jaw front was first removed with a round bur and the wound was closed using resorbable sutures. Histopathological examination revealed evidence of fibrous dysplasia in extensive fibrous marrow areas and irregularly shaped trabeculae (Figure 4). A further examination of the skeletal system was carried out that demonstrated no structural changes in other bone locations, so a monostotic progression form could be assumed. To rule out endocrine involvement, ultrasound of the thyroid gland and the gonads, as well as a blood serum analysis, was performed. The patient will be given annual radiographic follow-ups at our clinic.

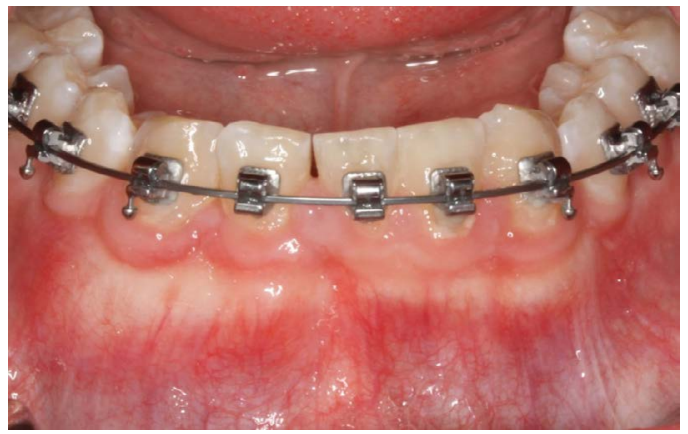

Figure 1: Clinical findings with a hard, non-compressive swelling in the right lower incisor area.

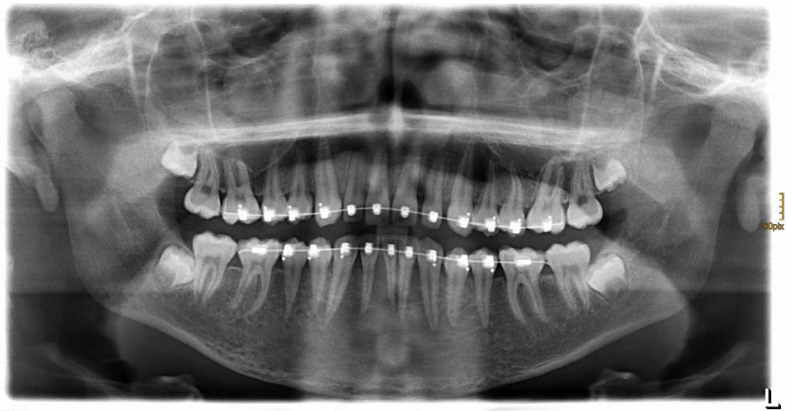

Figure 2: The orthopantomogram depicts a radiolucency in the apex region of the lower right incisors.

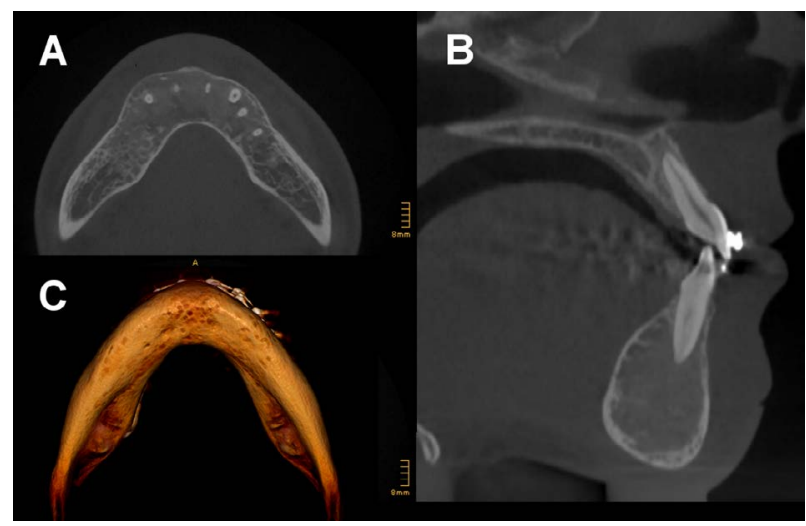

Figure 3: Horizontal $(A)$ and sagittal $(B)$ slices of the CBCT volume and 3D reconstruction $(C)$ with a "ground glass"-like appearance with mixed opacities and radiolucencies in the mental region in the sagittal and horizontal slices and a deformation of the caudal mandible visible in the $3 \mathrm{D}$ reconstruction.

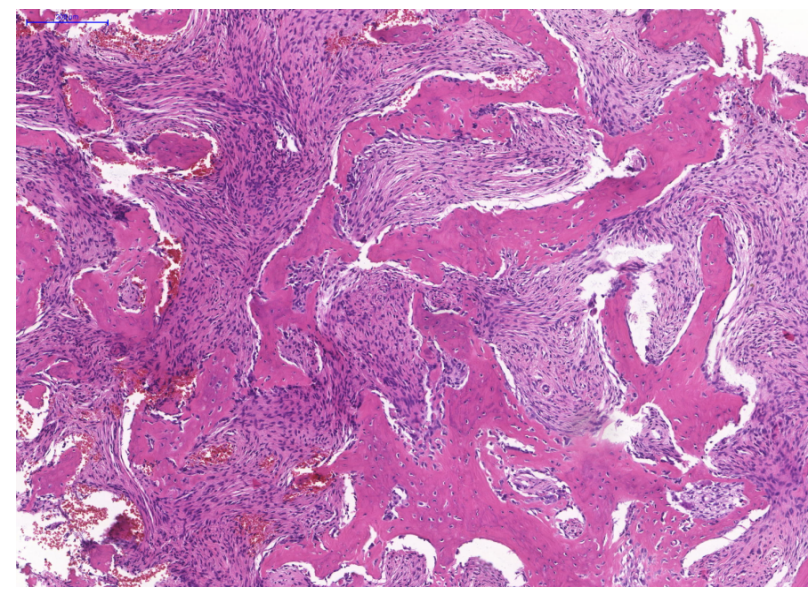

Figure 4: Hematoxylin-eosin staining with cell-rich fibrous stroma and woven irregular bone structures (10x).

\section{Patient 2}

A 47-year-old female patient was admitted with an uncertain osseous lesion of the left lower jaw. The bony lesion was first clinically noticeable following tooth extraction of the first molar in the left lower jaw. The general history of the patient revealed hypothyroidism, arterial hypertension, and neurodermatitis. Extraoral findings were inconspicuous, and the patient showed no neurological deficits in the facial area. Intraoral investigation showed an insufficiently maintained adult dentition. The teeth impressed inconspicuously, were not loosened, and were sensitive to cold temperature. Soft tissues remained free form irritation. In the former extraction area, an osseous protrusion could be felt on palpation.

CBCT demonstrated an irregularly shaped, sharply defined hypodense structure in the area of the lower jaw (Figure 5). The course of the nerve in this area was partially without bony limitation. Due to further localization at the base of the cranium, a computertomography (CT) was performed for further radiological clarification 10 days later.

The CT showed an expansive lesion with a "ground glass"-like internal structure and osteolyses spreading out from the sphenoid sinus. Significant multiple sclerosis and hyperplasia of the pars orbitalis 
of the ala major, as well as a complete displacement of the left sinus sphenoidalis with destruction of the corpus sphenoid. A characteristic milky, "ground glass" aspect was also detectable in the space between the lateral lamina and medial medulla of the ala major (Figure 6). In the lower jaw, a bony hyperplasia with a central osteolytic and a "ground glass"-like internal structure was found in the angulus area. The upper portions of the cervical spine included in the scan were regular. Because of the asymmetrical distribution and glassy aspect of the sphenoid and mandibular, a tentative diagnosis of fibrous dysplasia could be made radiologically. Paget's disease could not be excluded from the differential diagnosis.

A sample biopsy of the lower jaw was performed under local anesthesia. The histopathological findings confirmed the diagnosis of a benign lesion (Figure 7). Next-generation sequencing of the DNA detected a hotspot mutation of GNAS on exon 8 (R201H) which indicates the presence of fibrous dysplasia. The patient showed no endocrinopathies. Thus, a diagnosis of polyostotic progression was made. The patient was given follow-up checks every six months.

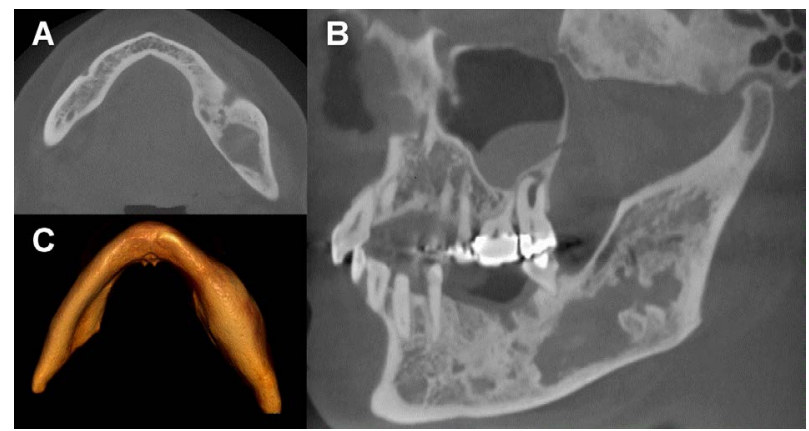

Figure 5: СВCT volume of patient 2 with horizontal $(A)$ and angulated sagittal (B) slices showing mixed heterogeneous sclerotic areas in the left mandibular angle. The 3D reconstruction $(\mathrm{C})$ depicts a bulging of mandible in the left molar region.

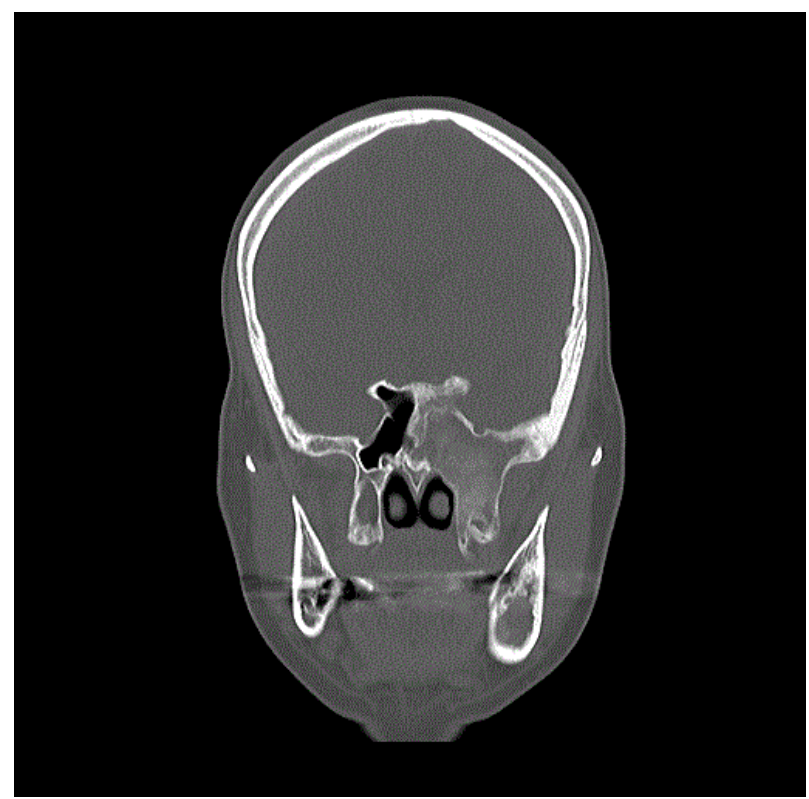

Figure 6: Coronal slice of the Computer tomography displaying the "ground glass"-like radiographic changes in the posterior maxilla and sinus region and the left mandible.

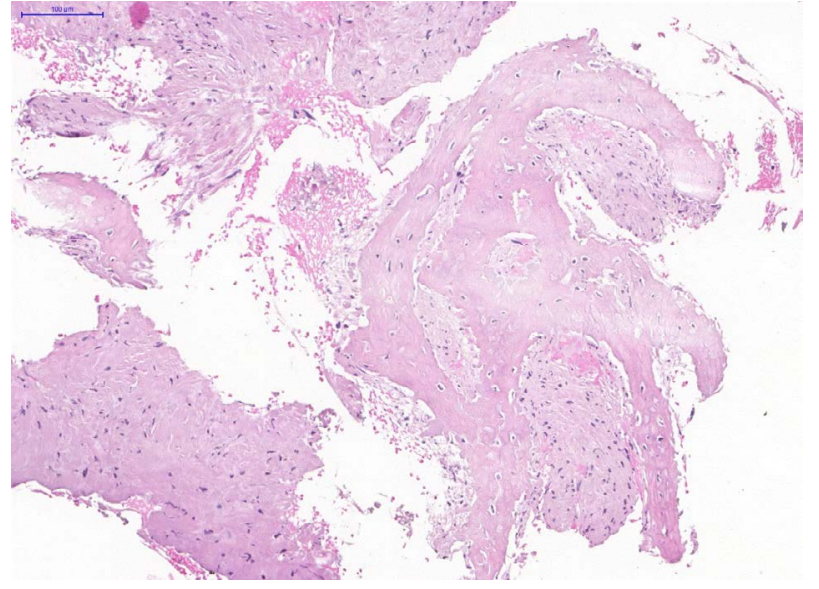

Figure 7: Histopathological findings using hematoxylin-eosin staining showing irregular formed bone fragments with spiky surfaces in a fibrous stroma resembling a "Chinese character" pattern (20x).

\section{Discussion}

Clinical development of FD is multiple, and varies according to the location and extent of the skeletal lesions. In the appendicular skeleton the disease often becomes apparent in older patients due to fractures, deformations, or pain [4]. Approximately $70 \%$ of patients with polyostotic fibrosis dysplasia and $10 \%$ of patients with the monostatic form have facial lesions [10]. The upper jaw is more frequently affected than the lower jaw and often demonstrates unilateral lesions in the posterior area $[4,11]$. In many cases involving lesions in the jaw area, the initial examination is accidental and occurs in the context of dental radiographic examinations in patients in the second and third decade of life [12]. This is due to the asymptomatic course of the disease, which is characterized by slow, mostly painless growth. Other progression forms display rapid growth with bone pain and paresthesia occurring in the region of the inferior alveolar nerve, but also a compression of the optic nerve when the orbit is involved. The lesions typically manifest in early childhood, often appearing as significant bony lesions as early as age 5 [13]. They display progressive growth in youth and less activity in adults due to the apoptosis of the bone marrow stroma cells [14]. A malignant transformation of the FD lesions is described in the literature in less than $1 \%$ of cases and is associated with the oncogenic potential of the GNAS mutation $[15,16]$.

Radiologically, a characteristic milky, "ground glass"-like radiolucency with alternating radio-opaque and radiolucent areas and thinned cortical bone appears. As the disease progresses, the lesions decrease in radioluence and reveal a heterogeneous appearance at focal cystic and sclerotic areas [17]. Ossifying fibroma, cemento-ossifying fibroma, cemento-osseous dysplasia, and giant cell carcinoma must be eliminated as differential diagnoses for this benign fibro-osseous lesion.

Currently, for FD treatment is no drug therapy available. Because the craniofacial form of FD is associated with bone pain in up to $40 \%$ of cases, bisphosphonates are often prescribed [18]. However, the indication of antiresorptive therapy should be carefully considered before risking necrosis of the jaw [19]. The indication for surgical therapy depends upon the individual lesion and the clinical behavior of that lesion. Lee et al. classify quiet, non-aggressive, and aggressive progression forms according to growth rates [17]. For slow-growing forms, regular clinical and radiographic follow-ups, as well as sensitive control of the local nerves, are sufficient. A biopsy is indicated in cases 
of atypical clinical behavior or the possibility of aggressive progression. Surgical therapy with recontouring of the facial bones can improve function and aesthetics. In cases of McCune-Albright's syndrome, endocrinological disorders should be threatened preoperatively, as high recurrence rates may occur. [20]. If possible, surgical therapy should be done after bone growth has been completed because the lesions will show less activity.

In general, conservative dental therapy can be performed safe in patients with FD. Regular clinical check-ups are recommended as the disease is associated with enamel hypoplasia, dentin dysplasia, taurodontism, an increased caries index score, and malocclusion [21]. Orthodontic therapy is described in the literature as more prolonged, but this is not a contraindication [22]. Dysgnathic surgery in patients with FD can also be performed in combination with contouring of the lesions or resection with stable results [23] and no detectable difference in the healing process [24]. In the current scientific literature, only case reports exist for implant placement in affected areas [25,26]. In the absence of evidence of osseous integration and inadequate data on long-term success, this procedure should therefore be reserved for exceptional cases.

\section{Conclusion}

This case report demonstrates the variable monostotic and polyostotic occurrences of fibrous dysplasia in the facial area. The variable radiological manifestation of the disease in different phases of life is notable. Fibrous dysplasia displays in a clinically and radiologically variable way, and therefore poses a challenge to the medical and dental practitioner. Therapy should always be determined by the progression of the disease and requires close interdisciplinary cooperation.

\section{References}

1. Boyce AM, Collins MT (1993) Fibrous Dysplasia/McCune-Albright Syndrome. In: GeneReviews(R). Seattle (WA): Seattle (WA): University of Washington, Seattle.

2. Shenker A, Weinstein LS, Moran A, Pescovitz OH, Charest NJ, et al. ( 1993) Severe endocrine and nonendocrine manifestations of the McCune-Albrigh syndrome associated with activating mutations of stimulatory $\mathrm{G}$ protein $\mathrm{Gs}$. J Pediatr 123: 509-518.

3. Dumitrescu CE, Collins MT (2008) McCune-Albright syndrome. Orphanet J Rare Dis 3: 12

4. Ricalde P, Magliocca KR, Lee JS (2012) Craniofacial fibrous dysplasia. J Oral Maxillofac Surg clin North Am 24: 427-441.

5. Lumbroso S, Paris F, Sultan C (2004) Activating Gsalpha mutations: analysis of 113 patients with signs of McCune-Albright syndrome--a European Collaborative Study. J Clin Endocrinol Metab 89: 2107-2113.

6. Weinstein LS, Liu J, Sakamoto A, Xie T, Chen M (2004) Minireview: GNAS: normal and abnormal functions. Endocrinology 145: 5459-5464.

7. Riminucci M, Saggio I, Robey PG, Bianco P (2006) Fibrous dysplasia as a stem cell disease. J Bone Miner Res 21: 125-131.

8. Riminucci M, Liu B, Corsi A, Shenker A, Spiegel AM, et al. (1999) The histopathology of fibrous dysplasia of bone in patients with activating mutations of the Gs alpha gene: site-specific patterns and recurrent histological hallmarks. J Pathol 187: 249-258.

9. Weinstein LS, Shenker A, Gejman PV, Merino MJ, Friedman E, et al. (1991) Activating mutations of the stimulatory $G$ protein in the McCune-Albright syndrome. N Engl J Med 325: 1688-1695.

10. Kim DD, Ghali GE, Wright JM, Edwards SP (2012) Surgical treatment of giant fibrous dysplasia of the mandible with concomitant craniofacial involvement. $J$ Oral Maxillofac Surg 70: 102-118.

11. Menon S, Venkatswamy S, Ramu V, Banu K, Ehtaih S, et al. (2013) Craniofacial fibrous dysplasia: Surgery and literature review. Ann Maxillofac Surg 3: 66-71.

12. Burke AB, Collins MT, Boyce AM (2016) Fibrous dysplasia of bone: craniofacial and dental implications. Oral Dis 2016.

13. Hart ES, Kelly MH, Brillante B, Chen CC, Ziran N, et al. (2007) Onset progression, and plateau of skeletal lesions in fibrous dysplasia and the relationship to functional outcome. J Bone Miner Res 22: 1468-1474.

14. Kuznetsov SA, Cherman N, Riminucci M, Collins MT, Robey PG, et al (2008) Age-dependent demise of GNAS-mutated skeletal stem cells and "normalization" of fibrous dysplasia of bone. J Bone Miner Res 23: 1731-1740.

15. Hoshi M, Matsumoto S, Manabe J, Tanizawa T, Shigemitsu T, et al. (2006) Malignant change secondary to fibrous dysplasia. Int J Clin Oncol 11: 229-235.

16. Ruggieri P, Sim FH, Bond JR, Unni KK (1994) Malignancies in fibrous dysplasia. Cancer 73: 1411-1424.

17. Lee JS, FitzGibbon EJ, Chen YR, Kim HJ, Lustig LR, et al. (2012) Clinica guidelines for the management of craniofacial fibrous dysplasia. Orphanet $\mathrm{J}$ Rare Dis 7: 2.

18. Kelly MH, Brillante B, Collins MT (2008) Pain in fibrous dysplasia of bone: agerelated changes and the anatomical distribution of skeletal lesions. Osteoporos Int 19: 57-63.

19. Metwally T, Burke A, Tsai JY, Collins MT, Boyce AM (2016) Fibrous Dysplasia and Medication-Related Osteonecrosis of the Jaw. J Oral Maxillofac Surg 74: 1983-1999.

20. Boyce AM, Burke A, Cutler Peck C, DuFresne CR, Lee JS, et al. (2016) Surgical Management of Polyostotic Craniofacial Fibrous Dysplasia: LongTerm Outcomes and Predictors for Postoperative Regrowth. Plast Reconst Surg 137: 1833-1839.

21. Akintoye SO, Lee JS, Feimster T, Booher S, Brahim J, et al. (2003) Dental characteristics of fibrous dysplasia and McCune-Albright syndrome. Oral Surg Oral Med Oral Pathol Oral Radiol Endod 96: 275-282.

22. Akintoye So, Boyce AM, Collins MT (2013) Dental perspectives in fibrous dysplasia and McCune-Albright syndrome. Oral Surg Oral Med Oral Pathol Oral Radiol Endod 116: 149-155.

23. Yeow VK, Chen YR (1999) Orthognathic surgery in craniomaxillofacial fibrous dysplasia. J Craniofac Surg 10: 155-159.

24. Cheung LK, Samman N, Pang M, Tideman H (1995) Titanium miniplate fixation for osteotomies in facial fibrous dysplasia--a histologic study of the screw/bone interface. Int J Oral Maxillofac Surg 24: 401-405.

25. Bajwa MS, Ethunandan M, Flood TR (2008) Oral rehabilitation with endosseous implants in a patient with fibrous dysplasia (McCune-Albright syndrome): a case report. J Oral Maxillofac Surg 66: 2605-2608.

26. Petrocelli M, Kretschmer W (2014) Conservative treatment and implant rehabilitation of the mandible in a case of craniofacial fibrous dysplasia: a case report. J Oral Maxillofac Surg 72: 901-906. 